\title{
Concurrent Exercise-Associated Ventricular Complexes and a Prolonged QT Interval are Associated with Evidence of Myocarditis
}

Michael Jeserich ${ }^{1 *}$, Bela Merkely ${ }^{2}$, Pascal Schlosser ${ }^{3}$, Simone Kimmel $^{4}$, Gabor Pavlik ${ }^{5}$, Stephan Achenbach ${ }^{1}$ and Jürgen Biermann ${ }^{6}$

${ }^{1}$ Department of Cardiology, Friedrich-Alexander-Universität Erlangen-Nürnberg, Erlangen, Germany

${ }^{2}$ Heart and Vascular Center, Semmelweis University, VároBudapest, Hungary

${ }^{3}$ Institute for Medical Biometry and Statistics, University of Freiburg, Germany

${ }^{4}$ Deaprtment of Medical Practice, Cardiology and Angiology, Nuernberg, Germany

${ }^{5}$ Department of Health Sciences and Sports Medicine, University of Physical Education, Budapest, Hungary

${ }^{6}$ Department for Cardiology and Angiology, Heart Center University of Freiburg, Germany

\begin{abstract}
Background: Precise myocardial tissue characterization in subjects with exercise-associated ventricular ectopy and prolonged QT interval but no obvious structural heart disease might facilitate further risk stratification. There is no data assessing QT intervals in patients with exercise-associated premature ventricular complexes (PVCs). Cardiovascular magnetic resonance (CMR) enables us to non-invasively assess myocardial scar and oedema. The purpose of our study was to assess the QT intervals in patients with exercise-associated premature ventricular complexes (PVCs).
\end{abstract}

Methods: We analyzed the QT and QTc intervals of the 162 consecutive patients with documented exerciseassociated PVCs but no history or evidence of coronary heart disease or cardiomyopathy we previously examined. Findings were compared with those from 182 new controls without exercise-associated PVCs matched for gender and age. Continuous 12-holder ECG during upright bicycle exercise test and ECG-triggered, T2-weighted, fast spin echo triple inversion recovery sequences and late gadolinium enhancement were obtained, as were LV function and dimensions.

Results: QT and QTc intervals were significantly prolonged in patients compared to controls $(396.3 \pm 33.5 \mathrm{~ms}$ vs. $385.9 \pm 28.4 \mathrm{~ms}, \mathrm{p}=0.002$ and $431.7 \pm 26.2 \mathrm{~ms}$ vs. $419.9 \pm 26.6 \mathrm{~ms}, \mathrm{p}=0.0001$ ). Eighty five percent of patients with exercise-associated PVCs and prolonged QT intervals had structural abnormalities in the myocardium suggestive of acute or remote myocarditis or myopericarditis.

Conclusion: Patients with exercise-associated PVCs showed prolonged QT intervals compared to controls. As a long QT interval is predictive of increased risk for arrhythmias or sudden cardiac death these findings may supply a potential mechanism for explaining the increased risk of some patients with exercise-associated PVCs.

Keywords: QT; QTc; ECG; Cardiovascular magnetic resonance imaging; Myocarditis; Premature ventricular complexes; Late gadolinium enhancement; STIR

Abbreviations: CMR: Cardiovascular Magnetic Resonance; ECG: Electrocardiogram; LV-EF: Left Ventricular Ejection Fraction; PVC's: Premature Ventricular Complexes; LV: Left Ventricular, Left Ventricle; LGE: Late Gadolinium Enhancement; STIR: Fast Spin Echo Triple Inversion Recovery Sequence (S)

\section{Introduction}

Exercise-associated premature ventricular complexes (PVCs) may be due to structural heart disease such as myocardial infarction, dilated or hypertrophic cardiomyopathy, arrhythmogenic right ventricular cardiomyopathy, or ion channel diseases [1-3]. In apparently healthy patients without underlying disease, the etiology of exercise-associated PVCs is often not revealed by basic non-invasive tests such as physical examinations, blood markers, echocardiography and/or treadmill exercise tests $[4,5]$.

During the past two decades, cardiovascular magnetic resonance (CMR) has evolved from a research tool to a clinically validated, safe and comprehensive imaging modality with good reproducibility and diagnostic accuracy superior to other non-invasive approaches [6]. Functional parameters of LV function, dimensions, and flow can be measured accurately $[7,8]$. Late gadolinium enhancement (LGE) and oedema sequences (STIR) have been performed in patients with acute or remote myocarditis, and oedema and midmyocardial or subepicardial LGE enhancement has been detected in patients with myocarditis $[6,9,10]$.

Moreover, QT prolongation has been documented in these patients with presumed myocarditis [11] and a greater QT dispersion was found in young patients with carditis [12]. A detailed analysis of QT and QTc intervals in patients with exercise-associated PVCs has not been published to date. We therefore compared the QT and QTc intervals of 162 consecutive patients we examined before and of whom $85 \%$ showed evidence of myocardial disease consistent with acute or previous myocarditis or myopericarditis [13] and compared these patients to a control group without PVCs. We hypothesized that exercise-associated PVCs due to remote or acute myocarditis may be associated with QT interval changes.

*Corresponding author: Michael Jeserich, Department of Cardiology, Friedrich-Alexander-Universität Erlangen-Nürnberg, Erlangen, Germany, Tel: 0049911209209; Fax: 00499112059962 E-mail: info@praxis-jeserich.de

Received December 26, 2017; Accepted January 08, 2018; Published January 10,2018

Citation: Jeserich M, Merkely B, Schlosser P, Kimmel S, Pavlik G, et al. (2018) Concurrent Exercise-Associated Ventricular Complexes and a Prolonged QT Interval are Associated with Evidence of Myocarditis. J Cardiovasc Dis Diagn 6: 305. doi: 10.4172/2329-9517.1000305

Copyright: (C) 2018 Jeserich M, et al.. This is an open-access article distributed under the terms of the Creative Commons Attribution License, which permits unrestricted use, distribution, and reproduction in any medium, provided the original author and source are credited. 


\section{Methods}

\section{Patients}

We analyzed retrospectively the 162 consecutive patients referred to our department for palpitations, thoracic pain or dyspnea who developed PVCs during exercise testing as reported before [13]. and compared them to 182 age- and sex-matched controls without exerciseinduced PVCs.

Patients and controls were excluded if they had a history or findings suggestive of coronary artery disease (history of myocardial infarction, signs of ischemia on stress tests, transmural scar on echocardiography, subendocardial or transmural scars on CMR in patients), dilated or hypertrophic cardiomyopathy, congenital heart disease, pulmonary hypertension, LV hypertrophy, significant valvular regurgitation or valvular stenosis, renal failure (creatinine $\geq 1.8 \mathrm{mg}$ / dl, GFR $<30$ ). Further exclusion factors were: chronic alcohol abuse, use of sympathomimetic drugs, Conn syndrome, chronic loop diuretic treatment, chronic use of laxatives or any other potential causes of hypokalemia.

Informed consent was obtained from each patient and control, and the study protocol conforms to the ethical guidelines of the 1975 Declaration of Helsinki as reflected in a priori approval by the institution's human research ethic committee. Our final cohort comprised 162 patients whom we compared to 182 age- and sexmatched controls. Exercise tests and echocardiography was performed in controls and patients. CMR was only performed in patients, not in controls. Echocardiography was used to confirm normal left ventricular function in controls. We did not perform exercise tests in patients with acute heart insufficiency and reduced LVEF $(\mathrm{EF}<50 \%)$, in line with acute fulminant myocarditis. All patients we examined were outpatients in stable conditions.

Eighty-five patients underwent a follow-up exercise test at a mean of 1.7 years ( 2 months to 6 years). They had been scheduled for a control visit after myocarditis. We conducted a subgroup analysis of QT intervals in this patient group. QT intervals were measured automatically over 10 heart beats during rest by custo cardio 100 and 200 with CUSTO software using the BAZETT formula (Custo Med GmbH, Ottobrunn, Germany).

To validate the automatic algorithm, we checked the QT measurements visually in 20 randomly chosen patients and observed good comparability. Automatic QT interval measured $388 \mathrm{~ms} \pm 29 \mathrm{~ms}$ vs. visually $384 \mathrm{~ms} \pm 26 \mathrm{~ms}, \mathrm{p}=0.9$. In 17 out of these 20 patients, the difference was $<10 \mathrm{~ms}$, in 15 patients $<5 \mathrm{~ms}$.

\section{Cardiovascular magnetic resonance (CMR)}

All images were acquired on a 1.5 Tesla magnetic resonance system (Intera CV 1.5T, Philips Medical Systems, Best, the Netherlands). A specifically designed software (Release 11 using a five-element cardiac phased-array coil combined with a homogeneity correction algorithm (Constant Level AppeaRance; CLEAR) was used [14]. Data acquisition was ECG-triggered. In addition to 2, 3 and 4-chamber long-axis views, 3-D short-axis volume data was acquired by steadystate free precession imaging to evaluate LV function and dimensions. Phase-contrast velocity images in the ascending aorta were obtained to measure stroke volume and rule out significant aortic insufficiency as described previously $[13,15,16]$. Functional and morphological data were evaluated using view forum 6.5. (Philips Medical Systems, Best, the Netherlands).
All patients but no controls underwent ECG-triggered, T2weighted, fast spin echo triple inversion recovery sequences (STIR) in a short axis view covering the whole left ventricle as described in our earlier studies $[13,15,16]$. Late gadolinium enhancement (LGE) imaging was obtained in all patients 10 minutes after iv administration of $0.2 \mathrm{mmol} / \mathrm{kg}$ gadolinium-diethylenetriaminepentaacetate using $3 \mathrm{D}$ inversion recovery turbo gradient echo sequences as described previously $[13,15,16]$.

\section{Statistical analysis}

Data are presented as means and standard deviation for quantitative variables and as absolute and relative frequencies for categorical variables. Variables between patients and controls were compared using t-tests for quantitative and Chi-square-tests for categorical variables. Correlations between quantitative patient characteristics were estimated by means of Pearson's correlation coefficient (r). All tests were two-sided and used a significance level of 0.05 to indicate statistical significance. Statistical analysis took place using SAS software (Statistical Analysis System) Version 9 and GraphPad Prism 5.01.

\section{Results}

Baseline characteristics of patients (with exercise-associated PVCs) and controls (without exercise-associated PVCs) are shown in Table 1. Controls did not differ significantly regarding age, sex and blood pressure. There was no significant difference in resting and maximum heart rate between patients and controls. Patients clinical characteristics were dyspnoea in $18 \%, 31 \%$ had thoracic pain, $43 \%$ fatigue and $60 \%$ palpitations. No patient or control had known long QT syndrome, catecholaminergic polymorphic ventricular tachycardia, early repolarisation syndrome or Brugada syndrome. Seven patients but no control had a history of paroxysmal atrial fibrillation and were in sinus rhythm during investigation; one patient presented permanent atrial fibrillation. As reported, the mean number of PVCs during exercise including the first minute after exercise was 29, range 1-240.

\section{Cardiovascular magnetic resonance (CMR) findings of patients}

As reported patients revealed normal LV function and dimensions (LVEF 63\% $\pm 8 \%$, LV stroke volume $97 \mathrm{ml} \pm 17 \mathrm{ml}$ and $\mathrm{LV}$ enddiastolic volume $155 \mathrm{ml} \pm 34 \mathrm{ml})$. Sixty out of $162(37 \%)$ patients displayed elevated T2-weighted CMR (STIR) values indicating oedema. Contrast enhancement was present in the myocardium of $110 / 162$ patients $(67.9 \%)$. Figures 1-3 depict an example of the CMR findings of a 57-year-old female patient. Twenty-seven percent of the patients presented locally-thickened pericardium $(\geq 4 \mathrm{~mm})$ and $17.9 \%$ with small pericardial effusion. Overall, structural anomalies had 139 (85.8\%) of those patients with exercise-associated PVCs.

\section{QT and QTc intervals}

QT and QTc intervals were significantly prolonged in patients

\begin{tabular}{|c|c|c|c|}
\hline Variable & Patients & Controls & p \\
\hline Mean age (years) & $57.4 \pm 12.8$ & $56.5 \pm 14.3$ & 0.56 \\
\hline Female $(\%)$ & $54.0 \pm 33.0$ & $78.0 \pm 39.6$ & 0.2 \\
\hline Weight $(\mathrm{kg})$ & $80.1 \pm 13.9$ & $80.0 \pm 14.5$ & 0.99 \\
\hline Systolic blood pressure $(\mathrm{mmHg})$ & $139.2 \pm 18.8$ & $143.4 \pm 19.0$ & 0.06 \\
\hline Diastolic blood pressure $(\mathrm{mmHg})$ & $82.5 \pm 10.1$ & $82.7 \pm 9.7$ & 0.88 \\
\hline Heart rate (beats per min) & $75.1 \pm 16.3$ & $74.7 \pm 11.2$ & 0.68 \\
\hline Maximal heart rate (beats per $\mathrm{min})$ & $140.2 \pm 24.4$ & $134.6 \pm 19.8$ & 0.59 \\
\hline
\end{tabular}

\pm : Standard Deviation

Table 1: Clinical characteristics. 


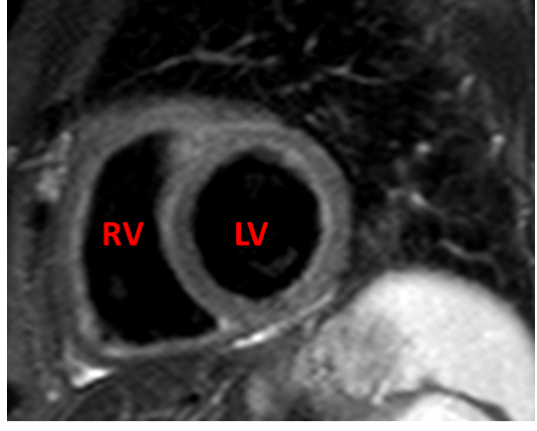

Figure 1: Depicts an example of the CMR findings of a 57-year-old female patient with fatigue, palpitations, new documented PVCs after an upper tract respiratory infect three weeks ago. Global STIR+ratio was elevated (2.26). Twochamber view. LV: Left Ventricle. RV: Right Ventricle. QT 425 ms, QTc 428 ms.

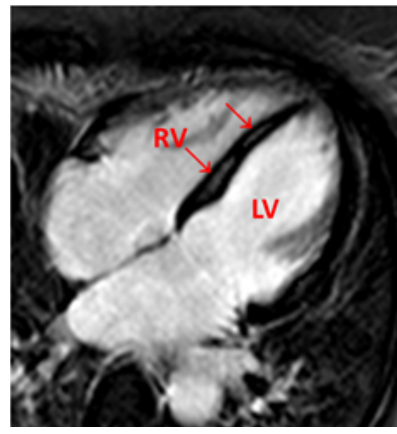

Figure 2: Late-enhancement image of the same patient. Note the midmyocardia enhancement in the septal $(\uparrow)$ wall. Four -chamber view. LV: Left Ventricle. RV: Right Ventricle.

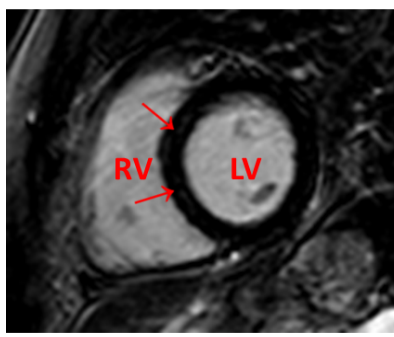

Figure 3: Two-chamber view of the same patient. the midmyocardial enhancement in the septal $(\uparrow)$ wall. LV: Left Ventricle. RV: Right Ventricle.

\begin{tabular}{|c|c|c|c|}
\hline Variable & Patients & Controls & p \\
\hline QT $(\mathrm{ms})$ & $396.3 \pm 33.5$ & $385.9 \pm 28.4$ & 0.002 \\
\hline QTc $(\mathrm{ms})$ & $431.7 \pm 26.2$ & $419.9 \pm 26.6$ & 0.0001 \\
\hline \multicolumn{2}{|c|}{ Male patients } & \multicolumn{2}{|c|}{ Female patients } \\
\hline QT (ms) & $397.3 \pm 35.0$ & $392.9 \pm 30.3$ & 0.44 \\
\hline QTc (ms) & $431.4 \pm 27.4$ & $431.9 \pm 24.2$ & 0.34 \\
\hline \multicolumn{2}{|c|}{ With oedema } & \multicolumn{2}{|c|}{ No oedema } \\
\hline QT (ms) & $399.5 \pm 31.4$ & $393.7 \pm 35.4$ & 0.30 \\
\hline QTc (ms) & $436.4 \pm 24.8$ & $429.6 \pm 27.0$ & 0.11 \\
\hline
\end{tabular}

ms: Milliseconds; \pm : Standard Deviation; With Edema: Patients with Edema; No Edema: Patients without Edema

Table 2: QT and QTc results.

compared to controls (Table 2) (Figure 4). QT and QTc intervals in patients with edema and without edema were not statistically different (Table 2) as were patients with or without scars. We observed no significant correlation between the QT or QTc intervals and the presence of edema or scar. There was no difference in male and female patients (Table 2). Thirty-three patients had a QTc interval $>450$ $\mathrm{ms}$, and two patients $>500 \mathrm{~ms}$. Although those two patients had no known channelopathies, we excluded them from a separate analysis. The difference in the QT and QTc intervals remained highly significant between patients and controls (QT $395.4 \mathrm{~ms} \pm 32.5 \mathrm{~ms}$ and QTc 430.5 $\mathrm{ms} \pm 24.3 \mathrm{~ms}, \mathrm{p}=0.005$ and 0.0002 ).

Eighty-five patients took a follow-up exercise test at a mean of 1.7 years (2 months to 6 years). In this patient subgroup, QT and QTc intervals at baseline were $397.9 \mathrm{~ms} \pm 34.5 \mathrm{~ms}$ and $428.5 \mathrm{~ms} \pm 25.0 \mathrm{~ms}$, and remained unchanged at follow-up with $399.5 \mathrm{~ms} \pm 42.5 \mathrm{~ms}$ and $429.5 \mathrm{~ms} \pm 50.8 \mathrm{~ms},(\mathrm{p}=0.78(\mathrm{QT})$ and $\mathrm{p}=0.87(\mathrm{QTc}))$.

There was no correlation between the amount of PVCs during exercise and QT or QTc in any of the patients $(\mathrm{p}=0.86)$ or in those with QTc below $500 \mathrm{~ms}(\mathrm{p}=0.87)$ or $450 \mathrm{~ms}(\mathrm{p}=0.83)$. Thus, the automatic QT measurement algorithm was not disturbed by the amount of PVCs.

\section{Discussion}

In the present study patients with exercise-associated PVCs exhibited a prolonged QT interval compared to controls. The vast majority of these patients presented myocardial edema, and midmyocardial or subepicardial scars consistent with acute or remote myocarditis [6,9-13]. A long QT interval is predictive of an increased risk for arrhythmias or sudden cardiac death [17]. Acute and long-term mortality in patients with viral myocarditis is higher in part due to sudden cardiac deaths caused by ventricular arrhythmias $[18,19]$, and there is evidence of QT prolongation in patients with acute myocarditis $[11,12]$. Some investigations of the prognostic significance of ventricular arrhythmia during exercise have assumed that PVCs are benign $[20,21]$ whereas others detected an association between PVCs and increased risk of cardiovascular or all-cause mortality rates [5,22-24]. In general, those studies examining patients with exercise-associated PVCs did not differentiate between the presence or high probability of coronary artery disease or other underlying cardiac diseases. As reported before [13], the vast majority of our patients with exercise-associated PVCs had structural anomalies consistent with acute or remote myocarditis, i.e. about two-thirds of them revealed subepicardial and/or midmyocardial late contrast-enhancement; about a third exhibited myocardial oedema typical of myocarditis $[6,9,10,25-28]$.

Prolonged QT intervals have been described in patients suffering from acute myocarditis [11,12]. Our novel finding of increased QT and QTc intervals in patients with exercise-associated PVCs compared to controls may supply a potential mechanism for explaining the increased risk of patients with PVCs reported in some studies. In addition, sudden cardiac death in athletes and the general population is not infrequently due to unrecognized myocarditis $[17,29]$. There is evidence that increased sympathetic nerve activity during physiologic

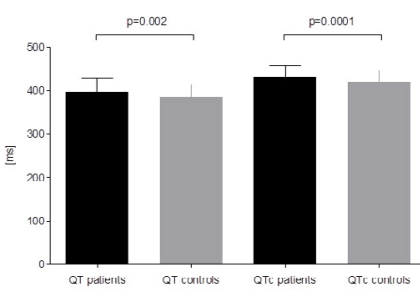

QT QTc

Figure 4: QT and QTc in patients and controls. QT and QTc were significantly increased in patients compared to controls. 
stress (e.g., exercise, swimming, emotion, loud noise) has a profound impact on the heart's contractile and electrical functions. In the predisposed heart, these stressors may lead to ventricular arrhythmias and sudden cardiac death [30]. In a case study with 26 patients with Williams-Beuren syndrome, a congenital developmental disorder, Collins et al. [31] reported a correlation between QTc, age, and PVCs at rest. Palatini et al. [32] found in 40 athletes a trend to higher prevalence of PVCs at rest compared to controls as well as prolonged QT and QTc intervals. Thus, there is evidence that patients with prolonged QT intervals and PVCs are at increased risk for lethal arrhythmias. To test the potential mechanisms, we compared the QT and QTc in patients with and without edema, and noted a tendency toward prolonged QT and QTc in the patients with edema.

We found no correlation between the presence of contrast enhancement and QT intervals. Other mechanisms for PVCs such as micro-reentry mechanisms without any detectable structural anomalities may therefore play an additional role. We found no difference in the QT and QTc intervals between male and female patients. QT intervals were unchanged in patients who came to a followup visit. In patients who presented initially with edema and came to a follow-up visit, the QTc interval was modestly but not significantly shortened. These findings are in line with potential contribution of an edema to prolonging the QT interval and to the findings that patients with takotsubo cardiomyopathy present with edema, negative $T$ waves, and QTc prolongation [33].

Structural abnormalities in terms of increased structural heterogeneity often constitute the basis for electrophysiological heterogeneity, e.g., with a prolonged QT interval and/or increased dispersion of repolarisation. In turn, these anomalies promote critical cardiac arrhythmias.

A limitation of our study is that only our patient group underwent CMR. The controls had no PVCs, no structural abnormalities on echocardiographic examination, normal LV function, and normal exercise tests. Therefore, it would not have been ethically justifiable to apply contrast agents to healthy volunteers. Thus, we cannot rule out that some controls might have exhibited areas of late contrastenhancement.

\section{Conclusion}

In conclusion, QT and QTc intervals were significantly prolonged in patients with exercise-associated premature ventricular beats and evidence of acute or remote myocarditis compared to controls. As a long QT interval is predictive of increased risk for arrhythmias or sudden cardiac death these findings may supply a potential mechanism for explaining the increased risk of some patients with exerciseassociated PVCs. These results add important information to what is currently known about the connection between structural and electrophysiological diseases of the heart.

\section{Acknowledgements}

We would like to thank Petra Nuß, Janina Wolfermann and Laura Fischer for collecting the data, Petra Nuß for typing parts of the manuscript, and Carole Cürten for proofreading.

\section{Funding Sources}

There was no funding specifically for this study.

\section{Conflict of Interest Statement}

On behalf of all authors, the corresponding author states that there is no conflict of interest.

\section{References}

1. Kadish A, Dyer A, Daubert JP, Quigg R, Estes NA, et al. (2004) Prophylactic defibrillator implantation in patients with nonischemic dilated cardiomyopathy. N Engl J Med 350: 2151-2158.

2. Adabag AS, Casey SA, Kuskowski MA, Zenovich AG, Maron BJ (2005) Spectrum and prognostic significance of arrhythmias on ambulatory Holter electrocardiogram in hypertrophic cardiomyopathy. J Am Coll Cardiol 45: 697-704.

3. Lehnart SE, Ackerman MJ, Benson DW Jr, Brugada R, Clancy CE, et al (2007) Inherited arrhythmias: A National Heart, Lung, and Blood Institue and Office of Rare Diseases workshop consensus report about the diagnosis, phenotyping, molecular mechanisms, and therapeutic approaches for primary cardiomyopathies of gene mutations affecting ion channel function. Circulation 116: $2325-2345$.

4. Jadhav A, Ingole A, Chockalingam A (2012) Ventricular ectopic beats: An overview of management considerations. Am J Med Sci 343: 150-154.

5. Morshedi-Meibodi A, Evans JC, Levy D, Larson MG, Vasan RS (2004) Clinical correlates and prognostic significance of exercise-induced ventricular premature beats in the community: The Framingham Heart Study. Circulation 109: $2417-2422$

6. Friedrich MG, Sechtem U, Holmvang G, Alakija P, Cooper LT, et al. (2009) International consensus group on cardiovascular magnetic resonance in myocarditis. Cardiovascular magnetic resonance in myocarditis: A JACC White Paper. J Am Coll Cardiol 53: 1475-1487.

7. Marcu CB, Beek AM, van Rossum AC (2006) Clinical applications of cardiovascular magnetic resonance imaging. CMAJ 175: 911-917.

8. Gibbons RJ, Araoz PA, Williamson EE (2009) The year in cardiac imaging. J Am Coll Cardiol 53: 54-70.

9. Abdel-Aty H, Boyé P, Zagrosek A, Wassmuth R, Kumar A, et al. (2005) Diagnostic performance of cardiovascular magnetic resonance in patients with suspected acute myocarditis: comparison of different approaches. J Am Coll Cardiol 45: 1815-1822.

10. Chu GC, Flewitt JA, Mikami Y, Vermes E, Friedrich MG (2013) Assessment of acute myocarditis by cardiovascular MR: Diagnostic performance of shortened protocols. Int J Cardiovasc Imaging 5: 1077-1083.

11. Ukena C, Mahfoud F, Kindermann I, Kandolf R, Kindermann M, et al. (2011) Prognostic electrocardiographic parameters in patients with suspected myocarditis. Eur J Heart Fail 13: 398-405.

12. Remigio de Aguiar MI, Saraiva LC, Santos CL (2010) QT Dispersion predicting acute rheumatic carditis. Cardiol Young 20: 473-476.

13. Jeserich M, Merkely B, Olschewski M, Kimmel S (2015) Patients with exerciseassociated ventricular ectopy present evidence of myocarditis. J Cardiovasc Magn Reson 17: 100.

14. Buerke B, Allkemper T, Kugel H, Bremer C, Evers S, et al. (2008) Qualitative and quantitative analysis of routinely postprocessed (CLEAR) CE-MRA data sets: are SNR and CNR calculations reliable? Acad Radiol 15: 1111-1117.

15. Jeserich M, Konstantinides S, Olschewski M, Pavlik G, Bode C, et al. (2010) Diagnosis of early myocardits in cardiovascular and gastrointestinal tract viral infection: Insights from cardiovascular magnetic resonance. Clin Res Cardiol 99: 707-714

16. Jeserich M, Brunner E, Kandolf R, Olschewski M, Kimmel S, et al. (2013) Diagnosis of viral myocarditis by cardiac magnetic resonance and viral genome detection in peripheral blood. Int J Cardiovasc Imaging 29: 121-129.

17. Maron BJ (2003) Sudden death in young athletes. N Engl J Med 349: 1064 1075.

18. Theleman KP, Kuiper JJ, Roberts WC (2001) Acute myocarditis (predominately lymphocytic) causing sudden death without heart failure. Am J Cardiol 88 1078-1083.

19. Kindermann I, Kindermann M, Kandolf R, Klingel K, Bültmann B, et al. (2008) Predictors of outcome in patients with suspected myocarditis. Circulation 118 639-648.

20. Dewey FE, Kapoor JR, Williams RS, Lipinski MJ, Ashley EA, et al. (2008) Ventricular arrhythmias during clinical treadmill testing and prognosis. Arch Intern Med 168: 225-234. 
Citation: Jeserich M, Merkely B, Schlosser P, Kimmel S, Pavlik G, et al. (2018) Concurrent Exercise-Associated Ventricular Complexes and a Prolonged QT Interval are Associated with Evidence of Myocarditis. J Cardiovasc Dis Diagn 6: 305. doi: 10.4172/2329-9517.1000305

Page 5 of 5

21. D’Ascenzi F, Zorzi A, Alvino F, Bonifazi M, Corrado D, et al. (2017) The prevalence and clinical significance of premature ventricular beats in the athlete. Scand J Med Sci Sports 27: 140-151.

22. Frolkis JP, Pothier CE, Blackstone EH, Lauer MS (2003) Frequent ventricular ectopy after exercise as a predictor of death. N Engl J Med 348: 781-790.

23. Kim J, Kwon M, Chang J, Harris D, Gerson MC, et al. (2016) Meta-analysis of prognostic implications of exercise-induced ventricular premature complexes in the general population. Am J Cardiol 118: 725-732.

24. Jouven X, Zureik M, Desnos $M$, Courbon D, Ducimetière $P(2000)$ Long-term outcome in asymptomatic men with exercise-induced premature ventricular depolarizations. N Engl J Med 343: 826-833.

25. Babu-Narayan SV, McCarthy KP, Ho SY, Magee AG, Kilner PJ, et al. (2007) Images in cardiovascular medicine. Myocarditis and sudden cardiac death in the young extensive fibrosis suggested by cardiovascular magnetic resonance in vivo and confirmed post mortem. Circulation 166: e122-125.

26. Karamitsos TD, Francis JM, Myerson S, Selvanayagam JB, Neubauer S (2009) The role of cardiovascular magnetic resonance imaging in heart failure. $\mathrm{J} A \mathrm{~m}$ Coll Cardiol 54: 1407-1424.

27. Assomull RG, Lyne JC, Keenan N, Gulati A, Bunce NH, et al. (2007) The role of cardiovascular magnetic resonance in patients presenting with chest pain, raised troponin and unobstructed coronary arteries. Eur Heart J 28: 1242-1249.

28. Gutberlet M, Spors B, Thoma T, Bertram H, Denecke T, et al. (2008) Suspected chronic myocarditis at cardiac MR: Diagnostic accuracy and association with immunohistologically detected inflammation and viral persistence. Radiology 246: 401-409.

29. Wren C, Sullivan JO, Wright C (2000) Sudden death in children and adolescents. Heart 83: 410-413.

30. Volders PG (2010) Novel insights into the role of the sympathetic nervous system in cardiac arrhythmogenesis. Heart Rhythm 7: 1900-1906.

31. Collins RT, Aziz PF, Swearingen CJ, Kaplan PB (2012) Relation of ventricular ectopic complexes to QTc interval on ambulatory electrocardiograms in Williams syndrome. Am J Cardiol 109: 1671-1676.

32. Palatini P, Maraglino G, Sperti G, Calzavara A, Libardoni M, et al. (1985) Prevalence and possible mechanisms of ventricular arrhythmias in athletes. Am Heart J 110: 560-567.

33. Perazzolo Marra M, Zorzi A, Corbetti F, De Lazzari M, Migliore F, et al. (2013) Apicobasal gradient of left ventricular myocardial edema underlies transien T-wave inversion and QT interval prolongation (Wellens' ECG pattern) in TakoTsubo cardiomyopathy. Heart Rhythm 10: 70-77. 